\title{
Three-level atom optics via the tunneling interaction
}

\author{
K. Eckert, ${ }^{1}$ M. Lewenstein, ${ }^{1}$ R. Corbalán, ${ }^{2}$ G. Birkl, ${ }^{3}$ W. Ertmer, ${ }^{3}$ and J. Mompart ${ }^{2}$ \\ ${ }^{1}$ Institute of Theoretical Physics, University of Hannover, Appelstrasse 2, D-30167 Hannover, Germany \\ ${ }^{2}$ Departament de Física, Universitat Autònoma de Barcelona, E-08193 Bellaterra, Spain \\ ${ }^{3}$ Institute of Quantum Optics, University of Hannover, Welfengarten 1, D-30167 Hannover, Germany
}

(Received 6 February 2004; published 19 August 2004)

\begin{abstract}
Three-level atom optics is introduced as a simple, efficient, and robust method to coherently manipulate and transport neutral atoms. The tunneling interaction among three trapped states allows us to realize the spatial analog of the stimulated Raman adiabatic passage, coherent population trapping, and electromagnetically induced transparency techniques and offers a wide range of possible applications. We investigate an implementation in optical microtrap arrays and show that under realistic parameters the coherent manipulation and transfer of neutral atoms among dipole traps could be realized in the millisecond range.
\end{abstract}

DOI: 10.1103/PhysRevA.70.023606

PACS number(s): 03.75.Be, 32.80.-t, 03.67.- a, 42.50.-p

\section{INTRODUCTION}

The coherent coupling between two orthogonal states of a quantum system gives rise to oscillations of their probability amplitudes such as the Rabi oscillations of a two-level atom interacting with a laser field. When three instead of two levels are considered, the interaction gives rise to a much richer phenomenology. A clear example is the electric-dipole interaction between a three-level atom and two laser modes, where a large number of techniques have been proposed and reported. These include the stimulated Raman adiabatic passage (STIRAP) method used to produce a complete population transfer between two internal quantum states of an atom or molecule [1], the modification of the optical properties of a medium by means of coherent population trapping (CPT) [2], and the electromagnetically induced transparency (EIT) [3] phenomena. All these three-level optics (TLO) techniques have been intensively studied with applications ranging from quantum control of atoms and molecules [1,4], laser cooling [5], and slowing down light to a few meters per second [6] to nonlinear optics with few photons [7]. Three-level systems in classical mechanics have been studied in [8].

In this paper, we introduce a set of tools analogous to the TLO techniques to efficiently and coherently manipulate and move atoms among traps and indicate some potential applications in interferometry, precision measurement, and quantum information. To illustrate the basic idea, let us start by considering two well-separated dipole traps and one single atom in, say, the left trap. As soon as the two traps are approached and tunneling takes place, the probability amplitude for the atom to be in the left or right trap oscillates in a Rabi-type fashion resembling a two-level atom in a coherent field. This tunneling-induced oscillation between the two traps can be used to coherently transfer atoms between traps and allows, in fact, for a simple realization of quantum computation [9]. However, this two-level technique is not robust under variations of the system parameters and requires precise control of distance and timing. The basic elements of the present proposal will be three traps and a single atom, with the atomic external degrees of freedom being controlled through the variation of the distance between each two traps. The proposed techniques do not require an accurate control of the system parameters and will be termed three-level atom optics (TLAO) techniques, since the interference of (single) atom matter waves is at the core of all these techniques.

\section{THREE-LEVEL ATOM OPTICS}

\section{A. Setup}

We will consider here arrays of optical microtraps where the dipole force of a red detuned laser field is used to store neutral atoms in each of the foci of a set of microlenses [10], although the TLAO can also be implemented in optical lattices and atomic waveguides. We will make use of two specific features of these arrays [11]: the possibility of an individual addressing of each trap and detecting whether a trap is occupied, and the independent displacement of columns or rows of microtraps. We assume here that we are able to initially store no or one atom per trap at will, as has been reported in single dipole traps [12] and in optical lattices [13]. Although we require only three traps, the use of trap arrays has the advantage of allowing several experiments to be done in parallel.

The three in-line dipole traps are modeled as three piecewise harmonic potentials of frequency $\omega_{x}$, and the neutral atom is assumed to be in the ground vibrational state of the left trap initially, while the other two traps are empty (Fig. 1). For simplicity, the temporal evolution of the distance between each of the two traps has been modeled with a cosine function truncated at the minimum separation. Then, the approach and eventual separation of the left and middle (middle and right) traps takes a time $t_{r}^{L M}\left(t_{r}^{M R}\right.$, here usually we will have $\left.t_{r}^{L M}=t_{r}^{M R} \equiv t_{r}\right)$, while $t_{i}^{L M}{ }^{r}\left(t_{i}^{M R}\right)$ is the time the traps remain at the minimum distance. The (unperturbed) three-level system is composed of the vibrational ground states of all three traps, i.e., $|0\rangle_{L},|0\rangle_{M}$, and $|0\rangle_{R}$, and the strength of the interaction between each of the two vibrational ground states is given (in the absence of the third trap) by the following tunneling "Rabi" frequency [14]:

$$
\frac{\Omega(\alpha d)}{\omega_{x}}=\frac{-1+e^{(\alpha d)^{2}}\{1+\alpha d[1-\operatorname{erf}(\alpha d)]\}}{\sqrt{\pi}\left(e^{2(\alpha d)^{2}}-1\right) / 2 \alpha d},
$$

where $\alpha d$ is the trap separation and $\alpha^{-1} \equiv \sqrt{\hbar / m \omega_{x}}$, with $m$ denoting the mass of the neutral atom. $\operatorname{erf}()$ is the error 


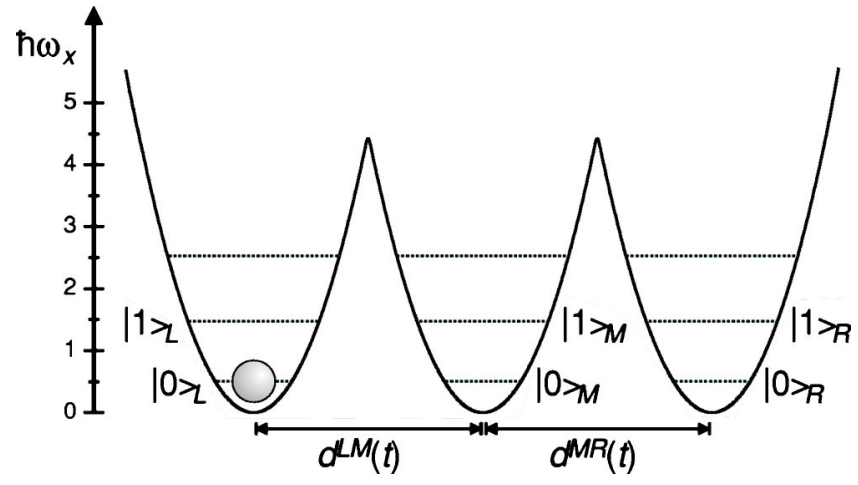

FIG. 1. Three-trap potential; $d^{L M}\left(d^{M R}\right)$ is the separation between left and middle (middle and right) traps. In the limit of a large separation $|n\rangle_{L},|n\rangle_{M},|n\rangle_{R}$ are the $n$th vibrational energy eigenstates of the corresponding single trap potentials.

function. The temporal shaping of $\Omega$ is realized by controlling the time dependence of $d(t)$. While Eq. (1) is useful to explore the analogies between TLO and TLAO, an exact treatment accounting for coupling to the excited vibrational states and direct coupling from the left to the right trap requires the integration of the Schrödinger equation. For our simulations, we assume a strong confinement in the $z$ direction, i.e., $\omega_{z} \gg \omega_{x}$, such that corresponding excitations can be neglected. In what follows, we will numerically integrate the one-dimensional (1D) Schrödinger equation to simulate the dynamics of the neutral atom in the three-trap potential, but we have checked consistency with the results of an integration of the 2D Schrödinger equation for the case $\omega_{x}=\omega_{y}$.

\section{B. Moving atoms between traps}

A robust method to coherently move atoms among traps using tunneling consists in extending the STIRAP technique [1]. The basic idea is to use the fact that one of the three eigenstates of the three-level system involves only the ground states of the two extreme traps,

$$
|D(\Theta)\rangle \equiv \cos \Theta|0\rangle_{L}-\sin \Theta|0\rangle_{R},
$$

where the mixing angle $\Theta$ is defined as $\tan \Theta \equiv \Omega^{L M} / \Omega^{M R}$ with $\Omega^{L M}\left(\Omega^{M R}\right)$ denoting the tunneling "Rabi" frequency between the left and middle (middle and right) traps. Following Eq. (2), it is possible to transfer the atom from $|0\rangle_{L}$ to $|0\rangle_{R}$ by adiabatically varying the mixing angle from $0^{\circ}$ to $90^{\circ}$, which means to approach and separate first the right trap to the middle trap and, with an appropriate delay, the left trap to the middle trap [Fig. 2(a)]. This counterintuitive sequence moves the atom directly from $|0\rangle_{L}$ to $|0\rangle_{R}$ with an almost negligible probability amplitude to be in the middle trap ground state [Fig. 2(b)].

As its optical analog, also this STIRAP-like process is robust against the variation of certain parameters. Figure 2(c) shows the transfer efficiency as a function of the time delay between the two trap approaches and of the amplitude $a_{\text {shake }}$ of shaking of the distance between the outer traps. Clearly, for a large range of delay times the transfer efficiency remains high. A similar robustness is present for variations of
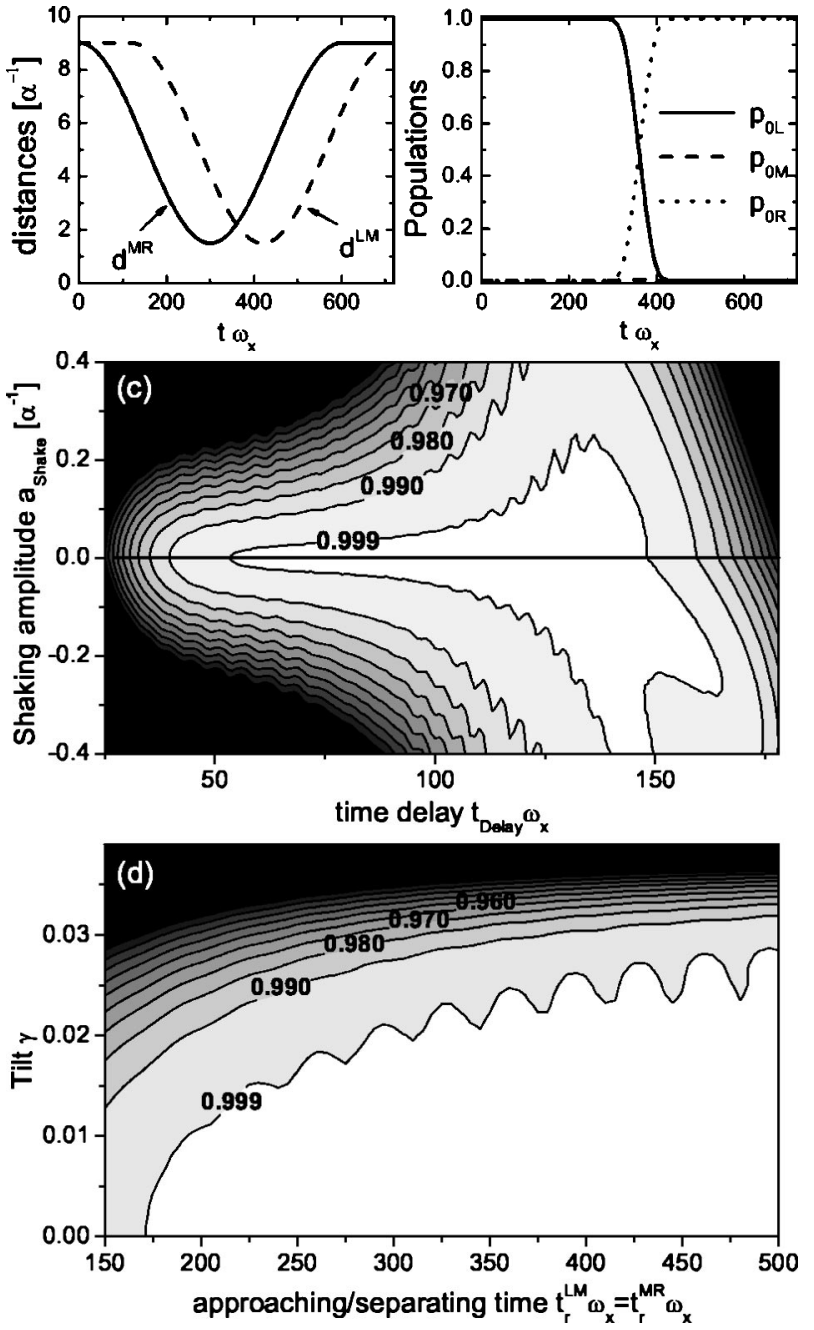

FIG. 2. (a) Approaching sequence for a STIRAP-like process, and (b) the corresponding ground-state populations; $d_{\max }^{L M} \alpha=d_{\max }^{M R} \alpha$ $=9, d_{\min }^{L M} \alpha=d_{\min }^{M R} \alpha=1.5, t_{r}^{L M} \omega_{x}=t_{r}^{M R} \omega_{x}=300, t_{i}^{L M} \omega_{x}=t_{i}^{M R} \omega_{x}=0$, and $t_{\text {delay }} \omega_{x}=120$. (c) Transfer efficiency from $|0\rangle_{L}$ to $|0\rangle_{R}$ as a function of the time delay $t_{\text {delay }}$ between the two trap approaches (horizontal axis) and of the amplitude of a shaking in the positions of the outer traps (vertical axis) with $\omega_{\text {shake }}=10^{-2} \omega_{x}$; all other parameters as above. For $a_{\text {shake }}>0$, the shaking of the outer traps is in phase; for $a_{\text {shake }}<0$ it is out of phase by $\pi$. (d) Transfer efficiency for an additional tilted potential $V_{\text {tilt }}(x)=\gamma \hbar \omega_{x} \alpha x$ parametrized by $\gamma$ as a function of the time $t_{r}$ needed to approach and separate the traps; all other parameters as in (b); $t_{\text {delay }}$ has been chosen as $t_{\text {delay }}=0.4 t_{r}$.

the maximum and minimum trap separation $d_{\max }$ and $d_{\min }$ and of $t_{r}$ and $t_{i}$ for each of the processes, provided that adiabaticity is maintained. The shaking of the traps provides an important source of decoherence for the experiment. We have assumed that the two outer traps shake with frequency $\omega_{\text {shake }}=10^{-2} \omega_{x}$, i.e., well below the trap frequency. If a transfer efficiency of 0.999 is desired, then for a proper choice of the delay time, shaking amplitudes of more than $5 \%$ of the minimal trap distance can be tolerated.

As another potential source of errors in the transfer process, we have analyzed a tilted potential, i.e., we have added a ramp of the form 


$$
V_{\text {tilt }}(x)=\gamma \hbar \omega_{x} \alpha x
$$

to the trapping potential, where the parameter $\gamma$ adjusts the slope of the ramp. For the parameters of our simulations, a value of $\gamma=0.01$ corresponds to a difference in the potential energy of $0.03 \hbar \omega_{x}$ between the outer traps at the minimal distance. As can be seen from Fig. 2(d), even for $\gamma \leqslant 0.02$ the transfer efficiency remains large as long as the time to approach and separate the traps is chosen long enough.

\section{Creation of a dark state}

Additionally, the approach sequence can be modified to create spatial superposition states with maximum atomic coherence, i.e., with $\left|c_{0 L} c_{0 R}^{*}\right|=1 / 2, c_{0 L}\left(c_{0 R}\right)$ being the probability amplitude to be in state $|0\rangle_{L}\left(|0\rangle_{R}\right)$. The basic idea is to adiabatically follow state (2) from $\Theta=0^{\circ}$ up to $\Theta=45^{\circ}$ by an appropriate delay in the approach processes and the subsequent simultaneous separation of the outermost traps [see Figs. 3(a) and 3(b) up to $\left.t \omega_{x}=780\right]$. The resulting state is the spatial equivalent to the well-known dark state arising in the CPT technique [2]. To prove that this state is dark, i.e., that it can be decoupled from the tunneling interaction, we approach and separate simultaneously the two extreme traps to the middle one [see Figs. 3(a) and 3(b) from $t \omega_{x}=780$ up to the end]. Clearly, the atom remains in the dark state in spite of the tunneling interaction.

The CPT process is robust with respect to the variation of external parameters as distances or timing, as long as the symmetry of the separation of the two traps is maintained. In Fig. 3(c), the influence of the choice of $t_{r}$ and of shaking is shown. If the outer traps shake out of phase by $\pi$, i.e., they move always in different directions such that the symmetry is not broken (the case $a_{\text {shake }}<0$ in the figure), then the transfer efficiency to the dark state is smaller but comparable to the STIRAP case. If they move in phase such that the symmetry is broken, then the efficiency is reduced, although in this case still some shaking can be tolerated for a large transfer efficiency.

Also in the presence of a tilted potential $V_{\text {tilt }}(x)$, cf., Eq. (3), as expected the efficiency of the creation of a superposition state drops faster than in the STIRAP case. In Fig. $3(d)$, the quantity

$$
p_{\text {split }}=\frac{\left|c_{0 L}\right|}{\sqrt{2}}+\frac{\left|c_{0 R}\right|}{\sqrt{2}}
$$

is plotted, i.e., the overlap with an equal superposition of the atom being in the ground state of the left and of the right trap, ignoring the phase between the two states (which is time-dependent due to the different energies of the left and the right ground state in the titled potential). Obviously the value of $\gamma$ that can be tolerated is an order of magnitude smaller than for the STIRAP-like process. At first sight, Fig. 3(d) seems counterintuitive, as the efficiency grows for a faster separation of the traps. To see the reason for this, consider first the easier case of splitting a single potential well into two $[15,16]$. If an atom is in the ground state of the initial trap, then for a very slow splitting it will remain in the ground state, which even for a slightly tilted potential is the
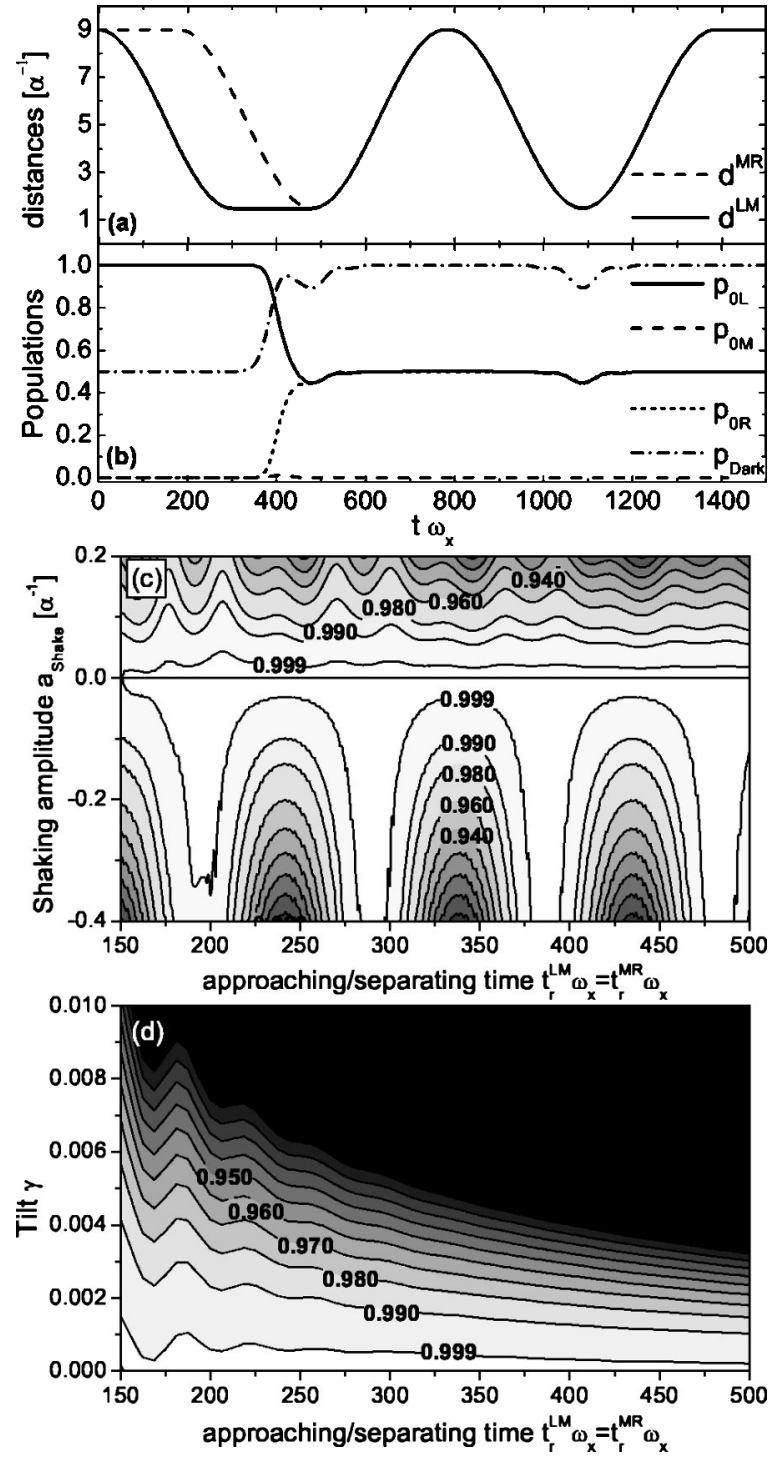

FIG. 3. (a) Approaching sequence for a CPT-like process; (b) ground state and dark state populations, $p_{\text {dark }}$ $=|\langle D(\Theta=\pi / 2) \mid \psi(t)\rangle|^{2}$; parameters as in Fig. 2 except for $t_{i}^{L M} \omega_{x}$ $=0, t_{i}^{M R} \omega_{x}=t_{\text {delay }} \omega_{x}=180$ for $t \omega_{x} \leqslant 780$ and $t_{i}^{M R} \omega_{x}=t_{\text {delay }} \omega_{x}=0$ for $t \omega_{x}>780$. (c) Dark state population $p_{\text {dark }}$ after the CPT process as a function of the time $t_{r}$ used to approach and separate the traps (horizontal axis) and of the amplitude $a_{\text {shake }}$ of a shaking in the trap positions (vertical axis). For $a_{\text {shake }}>0$, the shaking of the outer traps is in phase; for $a_{\text {shake }}<0$, it is out of phase by $\pi$; $\omega_{\text {shake }}$ $=10^{-2} \omega_{x}$ and all other parameters as above except for $t_{\text {delay }}=0.6 t_{r}$. (d) $p_{\text {split }}$ [see Eq. (4)] for an additional tilted potential as in Fig. 2(d) parametrized by $\gamma$ as a function of the time $t_{r}$ needed to approach and separate the traps; parameters as in (c).

trap with lower energy, such that the wave packet is no longer split between the wells $[15,17]$. For the CPT case, the process is similar, i.e., a very slow process puts the atom in a different state. As the evolution of the eigenvalues is more complicated, this does not necessarily have to be the state with lowest energy. For example, for the potentials and the parameters used here, the ground state of the left trap does evolve into the ground state of the middle trap for $t_{r} \rightarrow \infty$. 

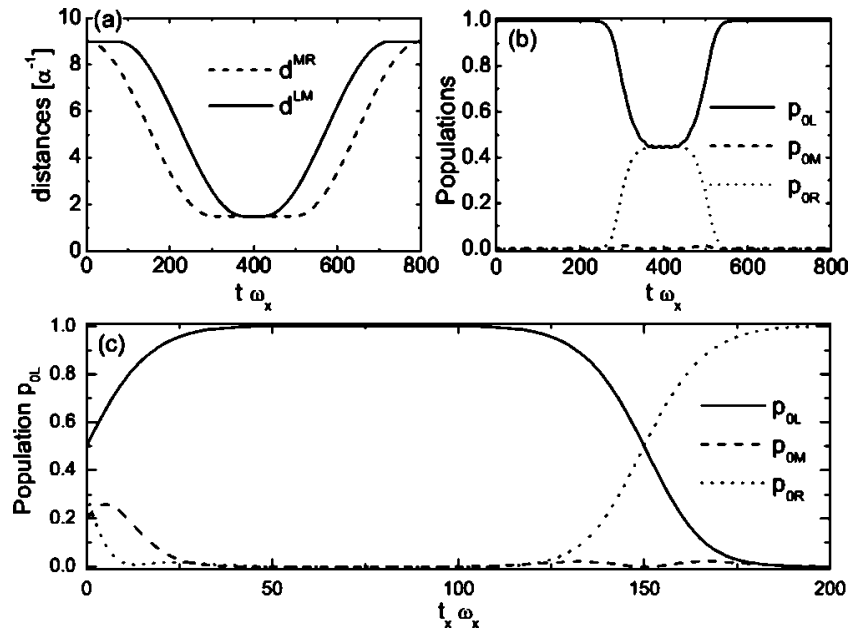

FIG. 4. (a) Approaching sequence for an EIT-like process, and (b) corresponding ground-state populations; $d_{\max }^{L M} \alpha=d_{\max }^{M R} \alpha=9$, $d_{\min }^{L M} \alpha=d_{\min }^{M R} \alpha=1.5, \quad t_{r}^{L M} \omega_{x}=t_{r}^{M R} \omega_{x}=300, \quad t_{i}^{L M} \omega_{x}=50, \quad t_{i}^{M R} \omega_{x}=200$, and $t_{\text {delay }} \omega_{x}=120$. (c) Ground-state populations as a function of the time delay.

The robust coherent splitting of the atomic wave function together with the possibility of individual trap manipulation anticipates applications in atomic interferometry. On the other hand, the sensitivity of the superposition dark states to dephasing [2] could be used in dipole trap systems to measure experimental imperfections such as uncorrelated shaking in the trap positions and/or intensity fluctuations of the trapping lasers.

\section{Inhibiting tunneling}

Finally, it is also possible to extend the EIT technique [3] to the atom optics case. The basic idea of EIT is to convert a medium that is opaque to a field resonant with a certain internal transition into one that is transparent by applying an intense driving field to an adjacent transition. In the threetrap system, we will inhibit the transition from $|0\rangle_{L}$ to $|0\rangle_{M}$ by driving the transition $|0\rangle_{M} \leftrightarrow|0\rangle_{R}$ via the tunneling interaction. Figures 4(a) and 4(b) show the inhibition of the $|0\rangle_{L}$ to $|0\rangle_{M}$ transition in spite of the proximity between left and middle traps. As in the STIRAP case, the plateau near the optimum delay evidences the robustness of the transition cancellation [Fig. 4(c)]. This atom optics EIT technique can create conditional phase shifts for quantum logic.

\section{E. Excited vibrational states}

It is worth noting that these techniques can also be applied to excited states, which relaxes the cooling requirements for the experimental setup. In Fig. 5, two examples for the threelevel system consisting of the first excited vibrational states of each trap are considered. Figure 5(a) shows the transfer of an atom from $|1\rangle_{L}$ to $|1\rangle_{R}$ via the STIRAP technique. Obviously there is a region (the dashed region in the figure) where even a robust simultaneous transfer of the two lowest states is possible. In Fig. 5(b), it is demonstrated that the atomic wave function can be coherently and equally split between

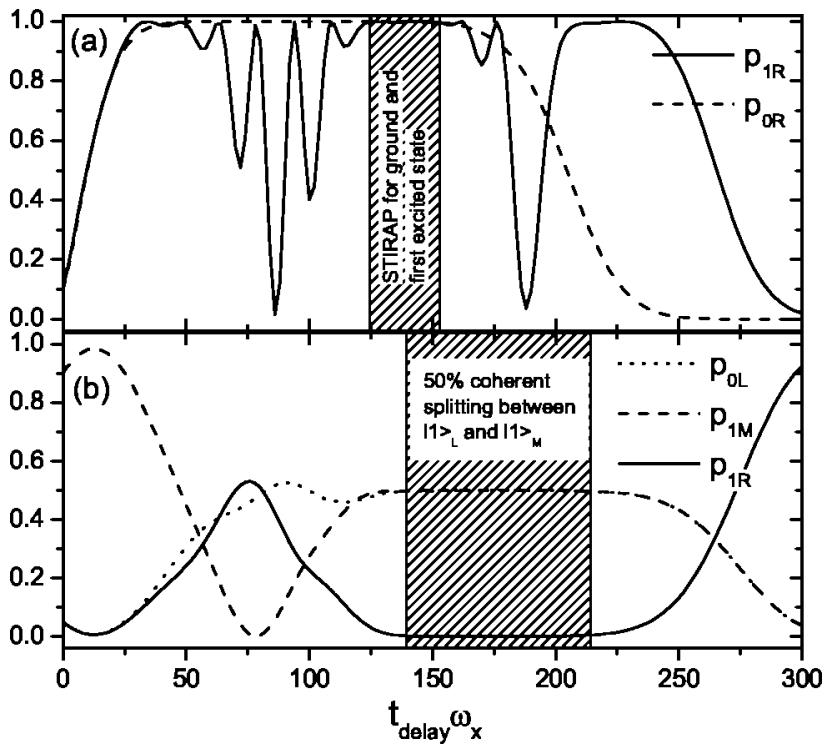

FIG. 5. Three-level atom optics for the first excited vibrational states: (a) transfer efficiency for STIRAP (dashed line: efficiency for the ground-state STIRAP for the same parameters); and (b) population for the coherent splitting between $|1\rangle_{L}$ and $|1\rangle_{M}$; the parameters are (a) $t_{r}^{L M} \omega_{x}=t_{r}^{M R} \omega_{x}=300, \quad t_{i}^{L M} \omega_{x}=t_{i}^{M R} \omega_{x}=0$, (b) $t_{r}^{L M} \omega_{x}=550, t_{r}^{M R} \omega_{x}=400, t_{i}^{L M} \omega_{x}=75, t_{i}^{M R} \omega_{x}=400$. In both cases $d_{\max }^{L M} \alpha=d_{\max }^{M R} \alpha=9$ and $d_{\min }^{L M} \alpha=d_{\min }^{M R} \alpha=1.5$.

the left and the middle trap (see the plateau around $t \omega_{x}$ $=180$ ). This effect, which is different from CPT and requires a combination of adiabatic and diabatic processes [18], is possible through a complicated variation of the dressed level structure of the first excited states when approaching the traps.

\section{PRACTICAL CONSIDERATIONS}

There are two important practical points for the implementation of the TLAO techniques in optical microtrap arrays: (i) the trapping frequencies must be the same for all microtraps and (ii) the approach process has to be adiabatic. The use of a single laser that illuminates simultaneously all microlenses assures the identity of all microtraps even under intensity fluctuations of the laser. In particular, typical trapping frequencies for microtrap arrays of ${ }^{87} \mathrm{Rb}$ atoms are $10^{5}-10^{6} \mathrm{~s}^{-1}$ in the transverse directions and $10^{4}-10^{5} \mathrm{~s}^{-1}$ along the laser beam direction [10,11], which means that the traps can be adiabatically approached in the millisecond range or even faster by using optimization techniques $[9,16]$. As we have shown, the STIRAP-like process works without the need for a precise control of timing and distances, it tolerates relatively large shaking amplitudes, and it is even robust with respect to differences in the ground-state energies of the three traps. For this reason, it is a promising method to move atoms among traps in order to create defectfree lattices or to shuttle around qubits for quantum computation. The spatial analog of the CPT technique also allows for inaccuracies in the parameters, but requires the separation process to be symmetric. However, under the influence of shaking with amplitudes of about $5 \%$ of the minimal trap 
distance, the dark state can still be populated with an efficiency of larger than 0.99 , which is a reasonable value for applications such as interferometry. This should be within reach of experiments since mechanical vibrations as the main source of shaking should mainly give rise to a correlated movement of all the traps.

Throughout the paper, we have assumed we would be able to cool down the atom to the lower vibrational states of the traps. In fact, sideband cooling to a temperature below $1 \mu \mathrm{K}$ with a ground-state population of $98.4 \%$ has been reported in optical lattices [19] with parameters very similar to the ones considered here. In this case, heating rates below $1 \mu \mathrm{K} / \mathrm{s}$ have been estimated [20]. In the presence of decoherence from heating, shaking, and spontaneous scattering, fidelities above 98\% [9] are expected for the ground-state TLAO techniques discussed here. However, it is worth noting again that all these techniques can also be applied to excited states. Note that the real trapping potentials differ from simple harmonic ones, but the adiabatic TLAO techniques do not rely on the particular shape of the trapping potentials.

\section{SUMMARY}

Summarizing, we have introduced a set of robust and efficient techniques to coherently manipulate and transport neutral atoms based on three-level atom optics. TLAO is the natural extension of the largely investigated STIRAP, CPT, and EIT techniques used in quantum optics [1-3] with the interaction mediated via tunneling and controlled by the shaping of the process of varying the separation between the traps. The fact that TLAO makes use of the tunneling interaction distinguishes it from the quantum optics case: the processes take place in the millisecond range, there are no dipole selection rules, and the use of excited states is possible. TLAO offers applications to atomic interferometry and quantum information; the possibility to use TLAO techniques for cooling, precision measurement, and lithography is presently being investigated. Some practical considerations for implementation in dipole trap arrays have been addressed, but these TLAO techniques are widely applicable to other atom optic systems such as magnetic microtraps, optical lattices, and dipole and magnetic waveguides, and to single ion traps.

\section{ACKNOWLEDGMENTS}

We would like to thank U. Poulsen, A. Sanpera, and F. Scharnberg for discussions. We acknowledge support from MCyT (Spain) and FEDER (EC) under project BFM200204369, and from the DGR (Catalunya) under project 2001SGR-187, as well as from CESCA-CEPBA, the DFG (SPP Quantum Information Processing and SFB 407), and from ACQP (EC).
[1] K. Bergmann, H. Theuer, and B. Shore, Rev. Mod. Phys. 70, 1003 (1998).

[2] E. Arimondo, in Progress in Optics, edited by E. Wolf (Elsevier Science, Amsterdam, 1996), Vol. 35, p. 257.

[3] S. E. Harris, Phys. Today 50(7), 36 (1997); J. P. Marangos, J. Mod. Opt. 45, 471 (1998).

[4] P. Marte, P. Zoller, and J. L. Hall, Phys. Rev. A 44, R4118 (1991).

[5] A. Aspect, E. Arimondo, R. Kaiser, N. Vansteenkiste, and C. Cohen-Tannoudji, Phys. Rev. Lett. 61, 826 (1988); G. Morigi, J. Eschner, and C. H. Keitel, ibid. 85, 4458 (2000); C. F. Roos, D. Leibfried, A. Mundt, F. Schmidt-Kaler, J. Eschner, and R. Blatt, ibid. 85, 5547 (2000).

[6] L. V. Hau, S. E. Harris, Z. Dutton, and C. H. Behroozi, Nature (London) 397, 594 (1999).

[7] M. D. Lukin and A. Imamoglu, Nature (London) 413, 277 (2001).

[8] P. R. Hemmer and M. G. Prentiss, J. Opt. Soc. Am. B 5, 1613 (1998); C. L. G. Alzar, M. A. G. Martinez, and P. Nussenzveig, Am. J. Phys. 70, 37 (2002).

[9] J. Mompart, K. Eckert, W. Ertmer, G. Birkl, and M. Lewenstein, Phys. Rev. Lett. 90, 147901 (2003).

[10] G. Birkl, F. B. J. Buchkremer, R. Dumke, and W. Ertmer, Opt. Commun. 191, 67 (2001).
[11] R. Dumke, M. Volk, T. Müther, F. B. J. Buchkremer, G. Birkl, and W. Ertmer, Phys. Rev. Lett. 89, 097903 (2002).

[12] D. Frese, B. Ueberholz, S. Kuhr, W. Alt, D. Schrader, V. Gomer, and D. Meschede, Phys. Rev. Lett. 85, 3777 (2000); N. Schlosser, G. Reymond, I. Protsenko, and P. Grangier, Nature (London) 411, 1024 (2001).

[13] M. Greiner, O. Mandel, T. Esslinger, T. W. Hänsch, and I. Bloch, Nature (London) 415, 39 (2002).

[14] This "Rabi" frequency corresponds to the splitting energy between the lowest symmetric and antisymmetric energy eigenstates.

[15] T. G. Tiecke, M. Kemmann, Ch. Buggle, I. Shvarchick, W. von Klitzing, and J. T. M. Walraven, J. Opt. B: Quantum Semiclassical Opt. 5, S119 (2003).

[16] W. Hänsel, J. Reichel, P. Hommelhoff, and T. W. Hänsch, Phys. Rev. A 64, 063607 (2001).

[17] F. Scharnberg et al. (unpublished).

[18] M. P. Fewell, B. Shore, and K. Bergmann, Aust. J. Phys. 50, 281 (1997).

[19] S. E. Hamann, D. L. Haycock, G. Klose, P. H. Pax, I. H. Deutsch, and P. S. Jessen, Phys. Rev. Lett. 80, 4149 (1998).

[20] V. Vuletić, C. Chin, A. J. Kerman, and S. Chu, Phys. Rev. Lett. 81, 5768 (1998). 